\title{
On the definition of solution to the total variation flow
}

\author{
Juha Kinnunen $^{1} \cdot$ Christoph Scheven ${ }^{2}$
}

Received: 10 June 2021 / Accepted: 30 October 2021 / Published online: 16 January 2022

(c) The Author(s) 2021

\begin{abstract}
We show that the notions of weak solution to the total variation flow based on the Anzellotti pairing and the variational inequality coincide under some restrictions on the boundary data. The key ingredient in the argument is a duality result for the total variation functional, which is based on an approximation of the total variation by area-type functionals.
\end{abstract}

Mathematics Subject Classification $35 \mathrm{~K} 67 \cdot 35 \mathrm{~K} 20 \cdot 35 \mathrm{~K} 92$

\section{Introduction}

This paper discusses the total variation flow

$$
\partial_{t} u-\operatorname{div}\left(\frac{D u}{|D u|}\right)=0 \quad \text { on } \Omega_{T}=\Omega \times(0, T),
$$

where $\Omega$ is a bounded domain in $\mathbb{R}^{n}$ and $T>0$. For this nonlinear parabolic equation we refer to the monograph by Andreu, Caselles and Mazon [5]. The total variation flow can be seen as the limiting case of the parabolic $p$-Laplace equation

$$
\partial_{t} u-\operatorname{div}\left(|D u|^{p-2} D u\right)=0, \quad 1<p<\infty,
$$

as $p \rightarrow 1$. A Sobolev space is the natural function space in the existence and regularity theories for a weak solution to the parabolic $p$-Laplace equation, see the monograph by DiBenedetto [12]. The corresponding function space for the total variation flow is functions of bounded variation and in that case the weak derivative of a function is a vector valued Radon measure. A standard definition of weak solution to the parabolic $p$-Laplace equation is based on integration by parts, but it is not immediately clear what is the corresponding

Communicated by Y. Giga.

\footnotetext{
Christoph Scheven

christoph.scheven@uni-due.de

Juha Kinnunen

juha.k.kinnunen@aalto.fi

1 Department of Mathematics, Aalto University, 11100 Aalto, Finland

2 Fakultät für Mathematik, Universität Duisburg-Essen, 45117 Essen, Germany
} 
definition of weak solution to the total variation flow. One possibility is to apply the socalled Anzellotti pairing [6]. This approach has been applied for the total variation flow, for example, in the monograph by Andreu, Caselles and Mazón [5].

For the parabolic $p$-Laplace equation, it is also possible to consider solutions to the parabolic variational inequality

$$
\frac{1}{p} \iint_{\Omega_{T}}|\nabla u|^{p} \mathrm{~d} x \mathrm{~d} t-\iint_{\Omega_{T}} u \partial_{t} \varphi \mathrm{d} x \mathrm{~d} t \leq \frac{1}{p} \iint_{\Omega_{T}}|\nabla u-\nabla \varphi|^{p} \mathrm{~d} x \mathrm{~d} t
$$

for every $\varphi \in C_{0}^{\infty}\left(\Omega_{T}\right)$. The variational approach goes back to Lichnewsky and Temam [17], who employed an analogous concept in the case of the time-dependent minimal surface equation. Wieser [18] showed that the variational approach gives the same class of weak solutions as the standard definition. Moreover, he introduced a more general class of quasiminimizers related to parabolic problems. The variational inequality related to the total variation flow is of the form

$$
\int_{0}^{T}\|D u(t)\|(\Omega) \mathrm{d} t-\iint_{\Omega_{T}} u \partial_{t} \varphi \mathrm{d} x \mathrm{~d} t \leq \int_{0}^{T}\|D(u-\varphi)(t)\|(\Omega) \mathrm{d} t
$$

for every $\varphi C_{0}^{\infty}\left(\Omega_{T}\right)$, where the total variation $\|D u(t)\|(\Omega)$ is a Radon measure for almost every $t \in(0, T)$. A distinctive feature is that the variational definition is based on total variation instead of weak gradient.

There are advantages in both approaches. For example, semigroup theory can be applied for the Anzellotti pairing and the direct methods in the calculus of variations can be applied in theory of parabolic variational integrals with linear growth. Initial and boundary value problems to the total variation flow have been studied by Andreu, Ballester, Caselles and Mazón [2,3] and by Andreu, Caselles, Díaz and Mazón [4]. They have shown that a unique solution exists to the problem

$$
\begin{cases}\partial_{t} u-\operatorname{div}\left(\frac{D u}{|D u|}\right)=0 & \text { in } \Omega \times(0, T), \\ u(x, t)=f(x) & \text { on } \partial \Omega \times(0, T), \\ u(x, t)=u_{o}(x) & \text { in } \Omega,\end{cases}
$$

where $\Omega$ is a bounded Lipschitz domain in $\mathbb{R}^{n}, f \in L^{1}(\partial \Omega)$ and $u_{0} \in L^{1}(\Omega)$. The case of homogeneous boundary data $f=0$ is discussed in [4] and [3] discusses inhomogeneous time-independent boundary data. The Neumann problem for the total variation flow has been studied in [2]. The concepts of solution discussed in [3] is more general than the concept of variational solution considered in this work. A variational approach to existence and uniqueness questions has been discussed by Bögelein, Duzaar and Marcellini [8], see also [11], and for the corresponding obstacle problem by Bögelein, Duzaar and Scheven [10]. A necessary and sufficient condition for continuity of a variational solution has been proved by DiBenedetto, Gianazza and Klaus [13]. Gianazza and Klaus [14] showed that variational solutions to the Cauchy-Dirichlet problem for the total variation flow are obtained as the limit as $p \rightarrow 1$ of variational solutions to the corresponding problem for the parabolic $p$-Laplace equation. See also Bögelein, Duzaar, Schätzler and Scheven [9].

Our main result in Theorem 5.1 below shows that the notions of weak solution to the total variation flow based on the Anzellotti pairing and the variational inequality coincide under natural assumptions. We consider weak solutions to a Cauchy-Dirichlet problem for the total 
variation flow, which can formally be written as

$$
\begin{cases}\partial_{t} u-\operatorname{div}\left(\frac{D u}{|D u|}\right)=0 & \text { in } \Omega_{T} \\ u=u_{o} & \text { on } \partial_{\mathcal{P}} \Omega_{T}\end{cases}
$$

For this problem, we consider the appropriate definitions of weak solution with timedependent boundary values, see Definitions 3.5 and 4.1 below. It is relatively straight forward to show that a weak solution to the total variation flow is a variational solution. This question has been studied in the context of metric measure spaces in [15]. However, it is much more challenging to prove that a variational solution is a weak solution. The key ingredient is a duality result for the total variation functional in Theorem 5.3. This is based on an approximation of the total variation by area-type functionals, see Theorem 5.2.

\section{Preliminaries}

\subsection{Functions of bounded variation}

Throughout this article, we consider a bounded Lipschitz domain $\Omega \subset \mathbb{R}^{n}$. We will prescribe Dirichlet boundary values on $\partial \Omega$ in form of solid boundary values. In the stationary case, this means that we choose an open Lipschitz domain $\Omega^{*} \subset \mathbb{R}^{n}$ with $\Omega \Subset \Omega^{*}$, consider the Dirichlet data

$$
u_{o} \in \mathrm{W}^{1,1}\left(\Omega^{*}\right) \cap L^{2}\left(\Omega^{*}\right),
$$

and restrict ourselves to functions that agree with $u_{o}$ almost everywhere with respect to the Lebesgue measure $\mathcal{L}^{n}$ on $\Omega^{*} \backslash \Omega$. We point out that in the parabolic setting, we will consider time-dependent boundary data as in (2.5), which satisfies (2.1) on almost every time slice. The space $\mathrm{BV}\left(\Omega^{*}\right)$ is defined as the space of functions $u \in L^{1}\left(\Omega^{*}\right)$ for which the distributional derivative $D u$ is given by a finite vector-valued Radon measure on $\Omega^{*}$. By $\|D u\|$ we denote the total variation measure of $D u$, which is defined by

$$
\|D u\|(A):=\sup \left\{\sum_{i=1}^{\infty}\left|D u\left(A_{i}\right)\right|: A_{i} \text { are pairwise disjoint Borel sets with } A=\bigcup_{i=1}^{\infty} A_{i}\right\}
$$

for every Borel set $A \subset \Omega^{*}$, cf. [1, Def. 1.4]. For the Dirichlet problem, we consider the function class

$$
\mathrm{BV}_{u_{o}}(\Omega):=\left\{u \in \operatorname{BV}\left(\Omega^{*}\right): u=u_{o} \text { a.e. in } \Omega^{*} \backslash \Omega\right\} \text {. }
$$

For $u \in \mathrm{BV}_{u_{o}}(\Omega)$, we write $D^{a} u$ and $D^{s} u$ for the absolutely continuous and the singular part of $D u$ with respect to the Lebesgue measure $\mathcal{L}^{n}$ and, moreover, $\nabla u$ for the RadonNikodym derivative of $D u$ with respect to the Lebesgue measure. With this notation, we have the decomposition

$$
D u=D^{a} u+D^{s} u=\nabla u \mathcal{L}^{n}+D^{s} u
$$

for every $u \in \mathrm{BV}_{u_{o}}(\Omega)$. From [1, Thm. 3.88] we know that on a bounded Lipschitz domain $\Omega \subset \mathbb{R}^{n}$, there exist bounded inner and outer trace operators

$$
T_{\Omega}: \mathrm{BV}(\Omega) \rightarrow L^{1}(\partial \Omega) \quad \text { and } \quad T_{\mathbb{R}^{n} \backslash \bar{\Omega}}: \mathrm{BV}\left(\mathbb{R}^{n} \backslash \bar{\Omega}\right) \rightarrow L^{1}(\partial \Omega) .
$$

With these trace operators, we have the following extension result for BV-functions. 
Lemma 2.1 ([1, Cor. 3.89]) Let $\Omega \subset \mathbb{R}^{n}$ be an open set with bounded Lipschitz boundary, $u \in \mathrm{BV}(\Omega)$ and $v \in \mathrm{BV}\left(\mathbb{R}^{n} \backslash \bar{\Omega}\right)$. Then the function

$$
w(x)=\left\{\begin{array}{l}
u(x), \text { for } x \in \Omega, \\
v(x), \text { for } x \in \mathbb{R}^{n} \backslash \bar{\Omega},
\end{array}\right.
$$

belongs to $\mathrm{BV}\left(\mathbb{R}^{n}\right)$, and its derivative is given by the measure

$$
D w=D u+D v+\left(T_{\mathbb{R}^{n} \backslash \bar{\Omega}} v-T_{\Omega} u\right) v_{\Omega} \mathcal{H}^{n-1}\llcorner\partial \Omega,
$$

where $v_{\Omega}$ denotes the generalized outer unit normal to $\Omega$. In the above formula, we interpret $D u$ and $D v$ as vector-valued measures on the entire $\mathbb{R}^{n}$ that are concentrated in $\Omega$ and in $\mathbb{R}^{n} \backslash \bar{\Omega}$, respectively.

We apply this lemma with the boundary values $u_{o}$ as in (2.1) in place of $v$. This is possible because we can extend the boundary values to a function $u_{o} \in W^{1,1}\left(\mathbb{R}^{n}\right)$ without changing the boundary condition.

\subsection{Parabolic function spaces}

A map $v:[0, T] \rightarrow X$ into a Banach space $X$ is called Bochner measurable or strongly measurable if it can be approximated by simple functions $v_{k}:[0, T] \rightarrow X$ in the sense $\left\|v_{k}(t)-v(t)\right\|_{X} \rightarrow 0$ for a.e. $t \in[0, T]$ as $k \rightarrow \infty$. A simple function is of the form

$$
v_{k}(t)=\sum_{i=1}^{N} v^{(i)} \chi_{E_{i}}(t)
$$

for $v^{(1)}, \ldots, v^{(N)} \in X$ and pairwise disjoint measurable sets $E_{1}, \ldots, E_{N} \subset[0, T]$. For $1 \leq$ $p \leq \infty$, we write $L^{p}(0, T ; X)$ for the space of equivalence classes of Bochner measurable functions $v:[0, T] \rightarrow X$ with $\|v(t)\|_{X} \in L^{p}([0, T])$.

For non-separable Banach spaces, the assumption of Bochner measurability often turns out to be too strong. For maps into non-separable dual spaces $X=X_{0}^{\prime}$, we use the following weaker condition. A function $v:[0, T] \rightarrow X_{0}^{\prime}$ is called weakly*-measurable if the map $[0, T] \ni t \mapsto\langle v(t), \varphi\rangle \in \mathbb{R}$ is measurable for every $\varphi \in X_{0}$, where $\langle\cdot, \cdot\rangle$ denotes the dual pairing between $X_{0}^{\prime}$ and $X_{0}$. Using this concept of measurability, we introduce the weak $*$-Lebesgue space

$$
L_{w *}^{p}\left(0, T ; X_{0}^{\prime}\right):=\left\{\begin{array}{l|l}
v:[0, T] \rightarrow X_{0}^{\prime} & \begin{array}{l}
v \text { is weakly*-measurable with } \\
t \mapsto\|v(t)\|_{X_{0}^{\prime}} \in L^{p}([0, T])
\end{array}
\end{array}\right\},
$$

with $1 \leq p \leq \infty$. The weak $*$-Lebesgue space with exponent $p=\infty$ naturally occurs in the case of the dual space $W^{-1, \infty}(\Omega)=\left[W_{0}^{1,1}(\Omega)\right]^{\prime}$, since

$$
\left[L^{1}\left(0, T ; W_{0}^{1,1}(\Omega)\right)\right]^{\prime}=L_{w *}^{\infty}\left(0, T ; W^{-1, \infty}(\Omega)\right),
$$

cf. [16, Sect. VII.4]. Moreover, from [1, Remark 3.12] we know that BV $\left(\Omega^{*}\right)$ is a dual space with a separable pre-dual $X_{0}$, whose elements take the form $g-\operatorname{div} G$ with $g \in C_{0}^{0}\left(\Omega^{*}\right)$ and $G \in C_{0}^{0}\left(\Omega^{*}, \mathbb{R}^{n}\right)$. We will consider the Cauchy-Dirichlet problem for the total variation flow with time-dependent boundary data satisfying

$$
u_{o} \in L^{1}\left(0, T ; W^{1,1}(\Omega)\right) \cap C^{0}\left([0, T] ; L^{2}(\Omega)\right) .
$$


The natural solution space for this problem is the weak*-Lebesgue space

$$
L_{w *}^{1}\left(0, T ; \mathrm{BV}_{u_{o}}(\Omega)\right):=\left\{u \in L_{w *}^{1}\left(0, T ; \mathrm{BV}\left(\Omega^{*}\right)\right): u=u_{o} \text { a.e. in }\left(\Omega^{*} \backslash \Omega\right)_{T}\right\} .
$$

The next lemma states that weak*-measurability implies measurability of the total variation functional.

Lemma 2.2 For $u \in L_{w *}^{1}\left(0, T ; \mathrm{BV}_{u_{o}}(\Omega)\right)$, the total variation $\|D u(t)\|(\bar{\Omega})$ depends measurably on $t \in(0, T)$.

Proof The total variation of the open set $\Omega^{*}$ is given by

$$
\|D u(t)\|\left(\Omega^{*}\right):=\sup \left\{\int_{\Omega^{*}} u(t) \operatorname{div} \zeta \mathrm{d} x \mid \zeta \in C_{0}^{1}\left(\Omega^{*}, \mathbb{R}^{n}\right),\|\zeta\|_{L^{\infty}\left(\Omega^{*}\right)} \leq 1\right\},
$$

cf. [1, Prop. 3.6]. The integrals in the supremum depend measurably on time by definition of the weak*-measurability. Since the supremum is taken over a separable set, the supremum is measurable as well. Therefore,

$$
\|D u(t)\|(\bar{\Omega})=\|D u(t)\|\left(\Omega^{*}\right)-\int_{\Omega^{*} \backslash \Omega}\left|\nabla u_{o}(t)\right| \mathrm{d} x
$$

depends measurably on $t \in(0, T)$.

\subsection{The area functional}

For a parameter $\mu \geq 0$, we consider the area functional

$$
\mathcal{A}_{u_{o}}^{(\mu)}(u):=\int_{\Omega} \sqrt{\mu^{2}+|\nabla u|^{2}} \mathrm{~d} x+\left\|D^{s} u\right\|(\bar{\Omega})
$$

where $u \in \mathrm{BV}_{u_{o}}(\Omega)$. The limit case $\mu=0$ corresponds to the total variation functional, i.e.

$$
\mathcal{A}_{u_{o}}^{(0)}(u)=\|D u\|(\bar{\Omega})
$$

for every $u \in \mathrm{BV}_{u_{o}}(\Omega)$. We point out that the functional $\mathcal{A}_{u_{o}}^{(\mu)}$ depends on the prescribed boundary function $u_{o}$. More precisely, since $\Omega$ is a bounded Lipschitz domain, Lemma 2.1 gives the decomposition

$$
D u=D u\left\llcorner\Omega+D u_{o}\left\llcorner\left(\Omega^{*} \backslash \bar{\Omega}\right)+\left(T_{\mathbb{R}^{n} \backslash \bar{\Omega}} u_{o}-T_{\Omega} u\right) v_{\Omega} \mathcal{H}^{n-1}\llcorner\partial \Omega\right.\right.
$$

for every $u \in \mathrm{BV}_{u_{o}}(\Omega)$. Therefore, the last term in (2.7) can be expressed as

$$
\begin{aligned}
\left\|D^{s} u\right\|(\bar{\Omega}) & =\left\|D^{s} u\right\|(\Omega)+\left\|D^{s} u\right\|(\partial \Omega) \\
& =\left\|D^{s} u\right\|(\Omega)+\int_{\partial \Omega}\left|T_{\Omega} u-T_{\mathbb{R}^{n} \backslash \bar{\Omega}} u_{o}\right| \mathrm{d} \mathcal{H}^{n-1},
\end{aligned}
$$

which implies

$$
\mathcal{A}_{u_{o}}^{(\mu)}(u)=\int_{\Omega} \sqrt{\mu^{2}+|\nabla u|^{2}} \mathrm{~d} x+\left\|D^{s} u\right\|(\Omega)+\int_{\partial \Omega}\left|T_{\Omega} u-T_{\mathbb{R}^{n} \backslash \bar{\Omega}} u_{o}\right| \mathrm{d} \mathcal{H}^{n-1} .
$$

The following approximation result for BV-functions will be useful for us.

Lemma 2.3 (Strict interior approximation) Let $\Omega \subset \mathbb{R}^{n}$ be a bounded Lipschitz domain and $u_{o} \in W^{1,1}\left(\Omega^{*}\right) \cap L^{2}\left(\Omega^{*}\right)$. For every $u \in \mathrm{BV}_{u_{o}}(\Omega)$ and $\mu \in[0,1]$, there exists a sequence of functions $u_{i} \in u_{o}+C_{0}^{\infty}(\Omega), i \in \mathbb{N}$, with $u_{i} \rightarrow u$ in $L^{2}(\Omega)$ and $\mathcal{A}_{u_{o}}^{(\mu)}\left(u_{i}\right) \rightarrow \mathcal{A}_{u_{o}}^{(\mu)}(u)$ as $i \rightarrow \infty$. 
Proof With the mollification operator $M_{\varepsilon}$ defined in $[9$, Sect.5] we define

$$
u_{i}=u_{o}+M_{\varepsilon_{i}}\left(u-u_{o}\right), \quad i \in \mathbb{N},
$$

for some sequence $\varepsilon_{i} \downarrow 0$ as $i \rightarrow \infty$. From [9, Lemma 5.1] we infer $u_{i} \rightarrow u$ in $L^{2}(\Omega)$ and $\mathcal{A}_{u_{o}}^{(1)}\left(u_{i}\right) \rightarrow \mathcal{A}_{u_{o}}^{(1)}(u)$ as $i \rightarrow \infty$. For the other parameters $\mu \in[0,1)$, the asserted convergence follows from the Reshetnyak continuity theorem [1, Thm. 2.39].

\section{The notion of weak solution by Anzellotti pairing}

For any vector field $z \in L^{\infty}\left(\Omega, \mathbb{R}^{n}\right)$ with $\operatorname{div} z \in L^{2}(\Omega)$, there exists a uniquely determined outer normal trace $[z, v] \in L^{\infty}(\partial \Omega)$ with

$$
\|[z, \nu]\|_{L^{\infty}(\partial \Omega)} \leq\|z\|_{L^{\infty}(\Omega)}
$$

and

$$
\int_{\Omega} w \operatorname{div} z \mathrm{~d} x+\int_{\Omega} z \cdot \nabla w \mathrm{~d} x=\int_{\partial \Omega}[z, v] w \mathrm{~d} \mathcal{H}^{n-1}
$$

for every $w \in W^{1,1}(\Omega) \cap L^{2}(\Omega)$, see [5, Prop.C.4]. We use the normal trace for the following version of an Anzellotti pairing, which is tailored for the Dirichlet problem.

Definition 3.1 For any $u \in \operatorname{BV}_{u_{o}}(\Omega) \cap L^{2}\left(\Omega^{*}\right)$ and $z \in L^{\infty}\left(\Omega, \mathbb{R}^{n}\right)$ with $\operatorname{div} z \in L^{2}(\Omega)$, we define the Anzellotti pairing of $z$ and $D u$ as the distribution

$$
(z, D u)_{u_{o}}(\varphi):=-\int_{\Omega} \operatorname{div} z u \varphi \mathrm{d} x-\int_{\Omega} z \cdot \nabla \varphi u \mathrm{~d} x+\int_{\partial \Omega}[z, \nu] u_{o} \varphi \mathrm{d} \mathcal{H}^{n-1}
$$

for every $\varphi \in C_{0}^{\infty}\left(\Omega^{*}\right)$.

It turns out that this distribution is a measure.

Lemma 3.2 For any $u \in \mathrm{BV}_{u_{o}}(\Omega) \cap L^{2}\left(\Omega^{*}\right)$ and $z \in L^{\infty}\left(\Omega, \mathbb{R}^{n}\right)$ with $\operatorname{div} z \in L^{2}(\Omega)$, the pairing $(z, D u)_{u_{o}}$ defines a Radon measure on $\bar{\Omega}$, and we have

$$
\left|(z, D u)_{u_{o}}(\bar{\Omega})\right| \leq\|z\|_{L^{\infty}(\Omega)}\|D u\|(\bar{\Omega}) .
$$

Before giving the proof, we state a variant of the preceding estimate that involves the area functional instead of the total variation. To this end, we note that for any vectors $z, v \in \mathbb{R}^{n}$ with $|z| \leq 1$ and $\mu>0$, we have the Fenchel-type inequality

$$
|z \cdot v| \leq \sqrt{\mu^{2}+|v|^{2}}-\mu \sqrt{1-|z|^{2}}
$$

This inequality can be verified by a straightforward calculation or by noting that $f_{\mu}^{*}(z)=$ $-\mu \sqrt{1-|z|^{2}}$ is the convex conjugate function of $f_{\mu}(v)=\sqrt{\mu^{2}+|v|^{2}}$ and recalling the general Fenchel inequality $|z \cdot v| \leq f_{\mu}(z)+f_{\mu}^{*}(v)$. We note that equality in (3.3) holds if and only if $z=v\left(\mu^{2}+|v|^{2}\right)^{-1 / 2}$. The Fenchel-type estimate above leads to an estimate for the Anzellotti pairing.

Lemma 3.3 For every $\mu \in(0,1]$, every $u \in \operatorname{BV}_{u_{o}}(\Omega) \cap L^{2}\left(\Omega^{*}\right)$ and every $z \in L^{\infty}\left(\Omega, \mathbb{R}^{n}\right)$ with $\|z\|_{L^{\infty}} \leq 1$ and $\operatorname{div} z \in L^{2}(\Omega)$ we have

$$
\left|(z, D u)_{u_{o}}(\bar{\Omega})\right| \leq \mathcal{A}_{u_{o}}^{(\mu)}(u)-\mu \int_{\Omega} \sqrt{1-|z|^{2}} \mathrm{~d} x .
$$


Proof of Lemmas 3.2 and 3.3 Let $u \in \mathrm{BV}_{u_{o}}(\Omega) \cap L^{2}\left(\Omega^{*}\right)$. Lemma 2.3 provides us with a sequence of approximating functions $u_{i} \in u_{o}+C_{0}^{\infty}(\Omega), i \in \mathbb{N}$, that converges strictly to $u$, i.e. $u_{i} \rightarrow u$ in $L^{2}(\Omega)$ and

$$
\left\|\nabla u_{i}\right\|_{L^{1}(\Omega)} \rightarrow\|D u\|(\bar{\Omega})
$$

as $i \rightarrow \infty$. For every test function $\varphi \in C_{0}^{\infty}\left(\Omega^{*}\right)$ we obtain

$$
\begin{aligned}
(z, D u)_{u_{o}}(\varphi) & =-\int_{\Omega} \operatorname{div} z u \varphi \mathrm{d} x-\int_{\Omega} z \cdot \nabla \varphi u \mathrm{~d} x+\int_{\partial \Omega}[z, \nu] u_{o} \varphi \mathrm{d} \mathcal{H}^{n-1} \\
& =\lim _{i \rightarrow \infty}\left(-\int_{\Omega} \operatorname{div} z u_{i} \varphi \mathrm{d} x-\int_{\Omega} z \cdot \nabla \varphi u_{i} \mathrm{~d} x\right)+\int_{\partial \Omega}[z, \nu] u_{o} \varphi \mathrm{d} \mathcal{H}^{n-1} \\
& =\lim _{i \rightarrow \infty} \int_{\Omega} z \cdot \nabla u_{i} \varphi \mathrm{d} x
\end{aligned}
$$

where in the last step, we applied (3.1) with $w=u_{i} \varphi$ and the fact $u_{i}=u_{o}$ on $\partial \Omega$ in the sense of traces. By (3.5), we deduce

$$
\begin{aligned}
\left|(z, D u)_{u_{o}}(\varphi)\right| & \leq \lim _{i \rightarrow \infty}\left\|\nabla u_{i}\right\|_{L^{1}(\Omega)}\|z\|_{L^{\infty}(\Omega)}\|\varphi\|_{C^{0}(\bar{\Omega})} \\
& =\|D u\|(\bar{\Omega})\|z\|_{L^{\infty}(\Omega)}\|\varphi\|_{C^{0}(\bar{\Omega})} .
\end{aligned}
$$

This implies that $(z, D u)_{u_{o}}$ defines a measure on $\bar{\Omega}$ that satisfies (3.2). This completes the proof of Lemma 3.2. For the proof of Lemma 3.3, we observe that according to Lemma 2.3, the sequence of approximating functions $u_{i} \in u_{o}+C_{0}^{\infty}(\Omega), i \in \mathbb{N}$, has the property $\mathcal{A}_{u_{o}}^{(\mu)}\left(u_{i}\right) \rightarrow \mathcal{A}_{u_{o}}^{(\mu)}(u)$ as $i \rightarrow \infty$ for every $\mu \in(0,1]$. We use (3.6) with a cut-off function $\varphi \in C_{0}^{\infty}\left(\Omega^{*}\right)$ with $\varphi \equiv 1$ on $\Omega$. Estimating the last integrand in (3.6) by means of (3.3), we arrive at

$$
\begin{aligned}
\left|(z, D u)_{u_{o}}(\bar{\Omega})\right| & =\left|(z, D u)_{u_{o}}(\varphi)\right| \\
& \leq \lim _{i \rightarrow \infty} \int_{\Omega} \sqrt{\mu^{2}+\left|\nabla u_{i}\right|^{2}} \mathrm{~d} x-\mu \int_{\Omega} \sqrt{1-|z|^{2}} \mathrm{~d} x \\
& =\mathcal{A}_{u_{o}}^{(\mu)}(u)-\mu \int_{\Omega} \sqrt{1-|z|^{2}} \mathrm{~d} x .
\end{aligned}
$$

This completes the proof of Lemma 3.3.

Next, we state an elementary identity for the Anzellotti pairings that will frequently be used in the proofs that follow.

Lemma 3.4 Let $z \in L^{\infty}\left(\Omega, \mathbb{R}^{n}\right)$ be with $\operatorname{div} z \in L^{2}(\Omega)$ and $v \in \mathrm{BV}_{u_{o}}(\Omega) \cap L^{2}(\Omega)$. Then we have

$$
(z, D v)_{u_{o}}(\bar{\Omega})-\int_{\Omega} z \cdot \nabla u_{o} \mathrm{~d} x=\int_{\Omega} \operatorname{div} z\left(u_{o}-v\right) \mathrm{d} x .
$$

Proof We use first the definition of the Anzellotti pairing and then property (3.1) of the normal trace with $w=u_{o}$ in order to have 


$$
\begin{aligned}
(z, D v)_{u_{o}}(\bar{\Omega}) & =-\int_{\Omega} v \operatorname{div} z \mathrm{~d} x+\int_{\partial \Omega}[z, v] u_{o} \mathrm{~d} \mathcal{H}^{n-1} \\
& =-\int_{\Omega} \operatorname{div} z\left(v-u_{o}\right) \mathrm{d} x-\int_{\Omega} u_{o} \operatorname{div} z \mathrm{~d} x+\int_{\partial \Omega}[z, v] u_{o} \mathrm{~d} \mathcal{H}^{n-1} \\
& =-\int_{\Omega} \operatorname{div} z\left(v-u_{o}\right) \mathrm{d} x+\int_{\Omega} z \cdot \nabla u_{o} \mathrm{~d} x .
\end{aligned}
$$

We apply the following definition of weak solution.

Definition 3.5 (Weak solution) Assume that $u_{o} \in L^{1}\left(0, T ; W^{1,1}(\Omega)\right) \cap C^{0}\left([0, T] ; L^{2}(\Omega)\right)$. We say that a function $u \in L_{w *}^{1}\left(0, T ; B V_{u_{o}}(\Omega)\right) \cap C^{0}\left([0, T] ; L^{2}(\Omega)\right)$ with $\partial_{t} u \in L^{2}\left(\Omega_{T}\right)$ is a weak solution of (1.1) if $u(0)=u_{o}(0)$ and if there exists a vector field $z \in L^{\infty}\left(\Omega_{T}, \mathbb{R}^{n}\right)$ with $\|z\|_{L^{\infty}} \leq 1, \operatorname{div} z=\partial_{t} u$ in $\Omega_{T}$ in the sense of distributions and

$$
\|D u(t)\|(\bar{\Omega})+\int_{\Omega \times\{t\}} \partial_{t} u(u-v) \mathrm{d} x=(z(t), D v)_{u_{o}}(\bar{\Omega})
$$

for every $v \in \mathrm{BV}_{u_{o}(t)}(\Omega) \cap L^{2}(\Omega)$ and a.e. $t \in(0, T)$.

We recall an equivalent way to formulate the preceding concept of solution that has already been observed in [4, Thm.1].

Lemma 3.6 A map $u \in L_{w *}^{1}\left(0, T ; B V_{u_{o}}(\Omega)\right) \cap C^{0}\left([0, T] ; L^{2}(\Omega)\right)$ with $\partial_{t} u \in L^{2}\left(\Omega_{T}\right)$ and $u(0)=u_{o}(0)$ is a weak solution of (1.1) in the sense of Definition 3.5 if and only if there exists a vector field $z \in L^{\infty}\left(\Omega_{T}, \mathbb{R}^{n}\right)$ with

$$
\operatorname{div} z=\partial_{t} u \text { in } \Omega_{T}
$$

in the sense of distributions, for which

$$
\|z\|_{L^{\infty}\left(\Omega_{T}\right)} \leq 1 \quad \text { and } \quad(z(t), D u(t))_{u_{o}}(\bar{\Omega})=\|D u(t)\|(\bar{\Omega})
$$

hold true for a.e. $t \in(0, T)$.

Remark 3.7 The condition (3.9) for the vector field $z$ can be interpreted as an analogue of the identity $z=\frac{D u}{|D u|}$ for BV-functions. In this sense, equation (3.8) is the generalization of the differential equation $(1.1)_{1}$ to the BV-setting.

Proof of Lemma 3.6 If $u$ is a weak solution of (1.1) in the sense of Definition 3.5, we simply choose $v=u(t)$ in (3.7) to deduce (3.9).

For the other direction, assume that $u \in L_{w *}^{1}\left(0, T ; B V_{u_{o}}(\Omega)\right) \cap C^{0}\left([0, T] ; L^{2}(\Omega)\right)$, with $\partial_{t} u \in L^{2}\left(\Omega_{T}\right)$, and $u(0)=u_{o}(0)$ and that there exists a vector field $z \in L^{\infty}\left(\Omega_{T}, \mathbb{R}^{n}\right)$ with the properties in (3.8) and (3.9). For $v \in \mathrm{BV}_{u_{o}(t)}(\Omega) \cap L^{2}(\Omega)$, we apply Lemma 3.4, once with $v$ and once with $u(t)$, to obtain

$$
\begin{aligned}
(z(t), D v)_{u_{o}}(\bar{\Omega}) & =\int_{\Omega} z(t) \cdot \nabla u_{o}(t) \mathrm{d} x+\int_{\Omega \times\{t\}} \partial_{t} u\left(u_{o}-v\right) \mathrm{d} x \\
& =(z(t), D u(t))_{u_{o}}(\bar{\Omega})+\int_{\Omega \times\{t\}} \partial_{t} u(u-v) \mathrm{d} x \\
& =\|D u(t)\|(\bar{\Omega})+\int_{\Omega \times\{t\}} \partial_{t} u(u-v) \mathrm{d} x,
\end{aligned}
$$

for a.e. $t \in(0, T)$. In the last line, we used (3.9). This proves that $u$ is a solution in the sense of Definition 3.5. 


\section{The concept of variational solution}

The following notion of solution of the Cauchy-Dirichlet problem (1.1) is based on the variational approach by Lichnewsky and Temam [17].

Definition 4.1 (Variational solution) Assume that the initial and boundary values satisfy $u_{o} \in L^{1}\left(0, T ; W^{1,1}(\Omega)\right) \cap C^{0}\left([0, T] ; L^{2}(\Omega)\right)$. A function $u \in L_{w *}^{1}\left(0, T ; B V_{u_{o}}(\Omega)\right) \cap$ $C^{0}\left([0, T] ; L^{2}(\Omega)\right)$ is called a variational solution of $(1.1)$ if

$$
\begin{aligned}
\int_{0}^{T}\|D u(t)\|(\bar{\Omega}) \mathrm{d} t & \leq \iint_{\Omega_{T}} \partial_{t} v(v-u) \mathrm{d} x \mathrm{~d} t+\int_{0}^{T}\|D v(t)\|(\bar{\Omega}) \mathrm{d} t \\
& -\frac{1}{2} \int_{\Omega}|v(T)-u(T)|^{2} \mathrm{~d} x+\frac{1}{2} \int_{\Omega}\left|v(0)-u_{o}(0)\right|^{2} \mathrm{~d} x
\end{aligned}
$$

holds true for every $v \in L_{w *}^{1}\left(0, T ; \mathrm{BV}_{u_{o}}(\Omega)\right) \cap C^{0}\left([0, T] ; L^{2}(\Omega)\right)$ with $\partial_{t} v \in L^{2}\left(\Omega_{T}\right)$.

In the following, we will consider variational solutions with the additional property $\partial_{t} u \in$ $L^{2}\left(\Omega_{T}\right)$, as it is required in the notion of weak solution in the sense of Definition 3.5. In this case, the variational inequality can also be considered separately on the time slices.

Lemma 4.2 Assume that $u_{o} \in L^{1}\left(0, T ; W^{1,1}(\Omega)\right) \cap C^{0}\left([0, T] ; L^{2}(\Omega)\right)$. A function $u \in$ $L_{w *}^{1}\left(0, T ; B V_{u_{o}}(\Omega)\right) \cap C^{0}\left([0, T] ; L^{2}(\Omega)\right)$, with $\partial_{t} u \in L^{2}\left(\Omega_{T}\right)$, is a variational solution of (1.1) in the sense of Definition 4.1 if and only if

$$
\|D u(t)\|(\bar{\Omega}) \leq \int_{\Omega \times\{t\}} \partial_{t} u(v-u) \mathrm{d} x+\|D v\|(\bar{\Omega})
$$

for a.e. $t \in(0, T)$ and every $v \in \mathrm{BV}_{u_{o}(t)}(\Omega) \cap L^{2}(\Omega)$ and if $u$ attains the initial values in the sense

$$
u(0)=u_{o}(0) \quad \text { in } L^{2}(\Omega) .
$$

Remark 4.3 Condition (4.2) can be reformulated in terms of the subdifferential

$$
\partial \Phi(u)=\left\{w \in L^{2}(\Omega): \Phi(u)+\langle w, v-u\rangle \leq \Phi(v) \text { for every } v \in L^{2}(\Omega)\right\}
$$

of the functional $\Phi: L^{2}(\Omega) \rightarrow \mathbb{R}$, defined by

$$
\Phi(u)= \begin{cases}\|D \bar{u}\|(\bar{\Omega}), & \text { if } \bar{u} \in \mathrm{BV}_{u_{o}}(\Omega) \cap L^{2}\left(\Omega^{*}\right), \\ \infty, & \text { if } \bar{u} \in L^{2}\left(\Omega^{*}\right) \backslash \mathrm{BV}_{u_{o}}(\Omega)\end{cases}
$$

Here, the function $\bar{u}$ denotes the extension of $u$ by $u_{o}$ to $\Omega^{*} \backslash \Omega$. By definition of the subdifferential, the variational inequality (4.2) can be reformulated as

$$
-\partial_{t} u(t) \in \partial \Phi(u(t)) \quad \text { for a.e. } t \in(0, T) .
$$

Proof of Lemma 4.2 Assume that the map $u$ satisfies (4.2) and (4.3) and let $v \in$ $L_{w *}^{1}\left(0, T ; \mathrm{BV}_{u_{o}}(\Omega)\right) \cap C^{0}\left([0, T] ; L^{2}(\Omega)\right)$ with $\partial_{t} v \in L^{2}\left(\Omega_{T}\right)$ be an arbitrary comparison function in (4.1). The function $v(t)$, for a.e. $t \in(0, T)$, is admissible in (4.2). Integrating the resulting inequalities over time, we deduce

$$
\int_{0}^{T}\|D u(t)\|(\bar{\Omega}) \mathrm{d} t \leq \iint_{\Omega_{T}} \partial_{t} u(v-u) \mathrm{d} x \mathrm{~d} t+\int_{0}^{T}\|D v(t)\|(\bar{\Omega}) \mathrm{d} t .
$$


Since $\partial_{t} u, \partial_{t} v \in L^{2}\left(\Omega_{T}\right)$ and $u(0)=u_{o}(0)$ by (4.3), an integration by parts implies

$$
\begin{aligned}
\iint_{\Omega_{T}} \partial_{t} u(v-u) \mathrm{d} x \mathrm{~d} t= & \iint_{\Omega_{T}} \partial_{t} v(v-u) \mathrm{d} x \mathrm{~d} t \\
& -\frac{1}{2} \int_{\Omega}|v(T)-u(T)|^{2} \mathrm{~d} x+\frac{1}{2} \int_{\Omega}\left|v(0)-u_{o}(0)\right|^{2} \mathrm{~d} x .
\end{aligned}
$$

Combining the two preceding formulae, we obtain (4.1), which proves that $u$ is a variational solution to (1.1).

For the opposite direction, we start with a variational solution $u \in L_{w *}^{1}\left(0, T ; \mathrm{BV}_{u_{o}}(\Omega)\right) \cap$ $C^{0}\left([0, T] ; L^{2}(\Omega)\right)$ with $\partial_{t} u \in L^{2}\left(\Omega_{T}\right)$. We begin with the observation that for any $v \in$ $\operatorname{BV}(\Omega) \cap L^{2}(\Omega)$, the extension

$$
\bar{v}(x, t)= \begin{cases}v(x), & x \in \Omega, \\ u_{o}(x, t), & x \in \Omega^{*} \backslash \Omega,\end{cases}
$$

defines a function $v \in L_{w *}^{1}\left(0, T ; \mathrm{BV}_{u_{o}}(\Omega)\right) \cap C^{0}\left([0, T] ; L^{2}\left(\Omega^{*}\right)\right)$ with $\partial_{t} v=0$ in $\Omega_{T}$. This follows by applying Lemma 2.1 separately on the time slices. For a cut-off function in time $\zeta \in C^{\infty}([0, T])$ with $\zeta(T)=0$ and the extension $\bar{v}$ defined above, we consider

$$
w=u+\zeta(t)(\bar{v}-u) .
$$

We note that this function is admissible as comparison function in (4.1), since the properties of $u$ and $\bar{v}$ imply $w \in L_{w *}^{1}\left(0, T ; \mathrm{BV}_{u_{o}}(\Omega)\right) \cap C^{0}\left([0, T] ; L^{2}(\Omega)\right)$ and $\partial_{t} w \in L^{2}\left(\Omega_{T}\right)$. By convexity of the total variation functional

$$
\|D w(t)\|(\bar{\Omega}) \leq(1-\zeta(t))\|D u(t)\|(\bar{\Omega})+\zeta(t)\|D \bar{v}(t)\|(\bar{\Omega})
$$

for a.e. $t \in(0, T)$, we obtain

$$
\begin{aligned}
\int_{0}^{T} \zeta(t)\|D u(t)\|(\bar{\Omega}) \mathrm{d} t \leq & \iint_{\Omega_{T}} \partial_{t}(u+\zeta(v-u))(v-u) \zeta \mathrm{d} x \mathrm{~d} t+\int_{0}^{T} \zeta(t)\|D \bar{v}(t)\|(\bar{\Omega}) \mathrm{d} t \\
& +\frac{1}{2} \int_{\Omega}\left|(1-\zeta(0)) u(0)+\zeta(0) v-u_{o}(0)\right|^{2} \mathrm{~d} x .
\end{aligned}
$$

Here, we also used the fact $\zeta(T)=0$, which ensures that no integral over the time slice at the final time occurs in the variational inequality. Integrating by parts, the integral involving the time derivative can be rewritten as

$$
\begin{aligned}
& \iint_{\Omega_{T}} \partial_{t}(u+\zeta(v-u))(v-u) \zeta \mathrm{d} x \mathrm{~d} t \\
& \quad=\iint_{\Omega_{T}} \partial_{t} u(v-u) \zeta \mathrm{d} x \mathrm{~d} t+\iint_{\Omega_{T}}\left(\zeta^{\prime} \zeta|v-u|^{2}+\zeta^{2} \frac{1}{2} \partial_{t}|v-u|^{2}\right) \mathrm{d} x \mathrm{~d} t \\
& \quad=\iint_{\Omega_{T}} \partial_{t} u(v-u) \zeta \mathrm{d} x \mathrm{~d} t-\frac{1}{2} \int_{\Omega} \zeta^{2}(0)|v-u(0)|^{2} \mathrm{~d} x
\end{aligned}
$$

Combining the preceding formulae, we arrive at

$$
\begin{aligned}
& \int_{0}^{T} \zeta(t)\|D u(t)\|(\bar{\Omega}) \mathrm{d} t \leq \iint_{\Omega_{T}} \partial_{t} u(v-u) \zeta \mathrm{d} x \mathrm{~d} t+\int_{0}^{T} \zeta(t)\|D \bar{v}(t)\|(\bar{\Omega}) \mathrm{d} t \\
& \quad+\frac{1}{2} \int_{\Omega}\left|(1-\zeta(0)) u(0)+\zeta(0) v-u_{o}(0)\right|^{2} \mathrm{~d} x-\frac{1}{2} \int_{\Omega} \zeta^{2}(0)|v-u(0)|^{2} \mathrm{~d} x .
\end{aligned}
$$


Our first goal is to show that the initial values are attained. To this end, we observe that an approximation argument implies the above inequality also for the characteristic function $\zeta=\chi_{[0, \tau]}$, for any $\tau \in(0, T)$. This gives

$$
\begin{aligned}
\int_{0}^{\tau}\|D u(t)\|(\bar{\Omega}) \mathrm{d} t \leq & \iint_{\Omega_{\tau}} \partial_{t} u(v-u) \mathrm{d} x \mathrm{~d} t+\int_{0}^{\tau}\|D \bar{v}(t)\|(\bar{\Omega}) \mathrm{d} t \\
& +\frac{1}{2} \int_{\Omega}\left(\left|v-u_{o}(0)\right|^{2}-|v-u(0)|^{2}\right) \mathrm{d} x
\end{aligned}
$$

for every $\tau \in(0, T)$. Since $\partial_{t} v=0$ in $\Omega_{T}$, an integration by parts gives

$$
\begin{aligned}
\iint_{\Omega_{\tau}} \partial_{t} u(v-u) \mathrm{d} x \mathrm{~d} t & =-\frac{1}{2} \iint_{\Omega_{\tau}} \partial_{t}|v-u|^{2} \mathrm{~d} x \mathrm{~d} t \\
& =\frac{1}{2} \int_{\Omega}|v-u(0)|^{2} \mathrm{~d} x-\frac{1}{2} \int_{\Omega}|v-u(\tau)|^{2} \mathrm{~d} x
\end{aligned}
$$

Combining the two preceding formulae, we arrive at

$$
\begin{aligned}
& \frac{1}{2} \int_{\Omega}|v-u(\tau)|^{2} \mathrm{~d} x+\int_{0}^{\tau}\|D u(t)\|(\bar{\Omega}) \mathrm{d} t \\
& \quad \leq \int_{0}^{\tau}\|D \bar{v}(t)\|(\bar{\Omega}) \mathrm{d} t+\frac{1}{2} \int_{\Omega}\left|v-u_{o}(0)\right|^{2} \mathrm{~d} x,
\end{aligned}
$$

for any $v \in \mathrm{BV}(\Omega) \cap L^{2}(\Omega)$ and $\tau \in(0, T)$. For a given $\varepsilon>0$, we choose $u_{o, \varepsilon} \in C_{0}^{\infty}(\Omega)$ with $\left\|u_{o, \varepsilon}-u_{o}(0)\right\|_{L^{2}(\Omega)} \leq \varepsilon$ and apply the preceding estimate with $v=u_{o, \varepsilon}$. Discarding the second integral on the left-hand side of (4.6), we have

$$
\frac{1}{2} \int_{\Omega}\left|u_{o, \varepsilon}-u(\tau)\right|^{2} \mathrm{~d} x \leq \int_{0}^{\tau}\left\|D \bar{u}_{o, \varepsilon}(t)\right\|(\bar{\Omega}) \mathrm{d} t+\frac{1}{2} \int_{\Omega}\left|u_{o, \varepsilon}-u_{o}(0)\right|^{2} \mathrm{~d} x,
$$

which implies

$$
\begin{aligned}
\frac{1}{4} \int_{\Omega}\left|u_{o}(0)-u(\tau)\right|^{2} \mathrm{~d} x & \leq \frac{1}{2} \int_{\Omega}\left|u_{o, \varepsilon}-u(\tau)\right|^{2} \mathrm{~d} x+\frac{1}{2} \int_{\Omega}\left|u_{o, \varepsilon}-u_{o}(0)\right|^{2} \mathrm{~d} x \\
& \leq \int_{0}^{\tau}\left\|D \bar{u}_{o, \varepsilon}(t)\right\|(\bar{\Omega}) \mathrm{d} t+\int_{\Omega}\left|u_{o, \varepsilon}-u_{o}(0)\right|^{2} \mathrm{~d} x \\
& \leq \int_{0}^{\tau}\left\|D \bar{u}_{o, \varepsilon}(t)\right\|(\bar{\Omega}) \mathrm{d} t+\varepsilon^{2}
\end{aligned}
$$

Using Lemma 2.1, we estimate the last integral by

$$
\int_{0}^{\tau}\left\|D \bar{u}_{o, \varepsilon}(t)\right\|(\bar{\Omega}) \mathrm{d} t \leq \tau \int_{\Omega}\left|\nabla u_{o, \varepsilon}\right| \mathrm{d} x+\int_{0}^{\tau} \int_{\partial \Omega}\left|T_{\mathbb{R}^{n} \backslash \bar{\Omega}} u_{o}(t)\right| \mathrm{d} \mathcal{H}^{n-1} \mathrm{~d} t \rightarrow 0
$$

as $\tau \downarrow 0$. Letting first $\tau \downarrow 0$ and then $\varepsilon \downarrow 0$ in (4.7), we conclude that

$$
\int_{\Omega}\left|u_{o}(0)-u(0)\right|^{2} \mathrm{~d} x=\lim _{\tau \downarrow 0} \int_{\Omega}\left|u_{o}(0)-u(\tau)\right|^{2} \mathrm{~d} x=0,
$$

which implies the assertion $u(0)=u_{o}(0)$.

It remains to show (4.2). For a cut-off function $\zeta \in C_{0}^{\infty}((0, T))$, inequality (4.5) and the fact that $u(0)=u_{o}(0)$ imply

$$
\int_{0}^{T} \zeta(t)\|D u(t)\|(\bar{\Omega}) \mathrm{d} t \leq \iint_{\Omega_{T}} \partial_{t} u(v-u) \zeta \mathrm{d} x \mathrm{~d} t+\int_{0}^{T} \zeta(t)\|D \bar{v}(t)\|(\bar{\Omega}) \mathrm{d} t
$$


for every $v \in \operatorname{BV}(\Omega) \cap L^{2}(\Omega)$, where $\bar{v}$ is defined by (4.4). For a given time $s \in(0, T)$ and $0<\delta<\min \{s, T-s\}$, we use this estimate with the cut-off function $\zeta(t)=\frac{1}{\delta} \phi\left(\frac{s-t}{\delta}\right)$, where $\phi \in C_{0}^{\infty}((-1,1))$ denotes a standard mollifier. By letting $\delta \downarrow 0$, we infer

$$
\|D u(s)\|(\bar{\Omega}) \leq \int_{\Omega \times\{s\}} \partial_{t} u(v-u) \mathrm{d} x+\|D \bar{v}(s)\|(\bar{\Omega})
$$

for a.e. $s \in(0, T)$ and every $v \in \mathrm{BV}(\Omega) \cap L^{2}(\Omega)$. This implies the remaining assertion (4.2) and completes the proof of Lemma 4.2.

\section{Equivalence of variational and weak solutions}

In this section we prove the equivalence of the two concepts of solution that have been introduced in Definition 3.5 and Definition 4.1, respectively. The precise statement of the result is the following.

Theorem 5.1 A function $u \in L_{w *}^{1}\left(0, T ; \mathrm{BV}_{u_{o}}(\Omega)\right) \cap C^{0}\left([0, T] ; L^{2}(\Omega)\right)$ with $\partial_{t} u \in L^{2}\left(\Omega_{T}\right)$ is a variational solution of (1.1) if and only if it is a weak solution of (1.1).

In Sect. 5.1 we show that a weak solution is a variational solution. The proof of the converse claim is presented in the remaining three subsections. The key step is an elliptic duality result for the total variation functional in Sect. 5.3. This will be established as a stability result by approximating the total variation by area-type functionals in Sect. 5.2. Finally, in Sect. 5.4 we complete the proof of the claim that a variational solution is a weak solution.

\subsection{Weak solutions are variational solutions}

Assume that $u \in L_{w *}^{1}\left(0, T ; \mathrm{BV}_{u_{o}}(\Omega)\right) \cap C^{0}\left([0, T] ; L^{2}(\Omega)\right)$, with $\partial_{t} u \in L^{2}\left(\Omega_{T}\right)$, is a weak solution according to Definition 3.5. Let $z \in L^{\infty}\left(\Omega_{T}\right)$ with $\|z\|_{L^{\infty}} \leq 1$ be the vector field that is provided by Definition 3.5. For a.e. $t \in(0, T)$ and any $v \in \mathrm{BV}_{u_{o}(t)}(\Omega) \cap L^{2}(\Omega)$, we use (3.7) and (3.2) to deduce

$$
\|D u(t)\|(\bar{\Omega})+\int_{\Omega \times\{t\}} \partial_{t} u(u-v) \mathrm{d} x=(z(t), D v)_{u_{o}}(\bar{\Omega}) \leq\|D v\|(\bar{\Omega})
$$

for a.e. $t \in(0, T)$. This means that the variational inequality (4.2) is satisfied on a.e. time slice, and Lemma 4.2 implies that $u$ is a variational solution according to Definition 4.1.

\subsection{An auxiliary result for the area functional}

The following approximation result for the area functional (2.7) will be applied in the proof of Theorem 5.3.

Theorem 5.2 Let $f \in W^{-1, \infty}(\Omega) \cap L^{2}(\Omega), u_{o} \in W^{1,1}(\Omega) \cap L^{2}(\Omega), \mu>0$, and $\lambda \in \mathbb{R}$ be given. Assume that $u \in \mathrm{BV}_{u_{o}}(\Omega) \cap L^{2}\left(\Omega^{*}\right)$ is a minimizer of the functional

$$
\Psi(v)=\mathcal{A}_{u_{o}}^{(\mu)}(v)+\int_{\Omega}\left(\frac{\lambda}{2}|v|^{2}+f\left(v-u_{o}\right)\right) \mathrm{d} x
$$


in the space $\mathrm{BV}_{u_{o}}(\Omega) \cap L^{2}\left(\Omega^{*}\right)$. Then the vector field

$$
z=\frac{\nabla u}{\sqrt{\mu^{2}+|\nabla u|^{2}}}
$$

satisfies

$$
\operatorname{div} z=\lambda u+f \text { in } \Omega
$$

in the sense of distributions and we have the estimate

$$
\begin{aligned}
& \mathcal{A}_{u_{o}}^{(\mu)}(u)+\int_{\Omega}\left(\frac{\lambda}{2}|u|^{2}+f\left(u-u_{o}\right)\right) \mathrm{d} x \\
& \quad \leq \int_{\Omega} z \cdot \nabla u_{o} \mathrm{~d} x+\frac{\lambda}{2} \int_{\Omega}\left|u_{o}\right|^{2} \mathrm{~d} x+\mu \int_{\Omega} \sqrt{1-|z|^{2}} \mathrm{~d} x .
\end{aligned}
$$

Proof For the proof of (5.1), we test the minimality of $u$ with the comparison map $v_{r}=$ $u-r \varphi \in \mathrm{BV}_{u_{o}}(\Omega) \cap L^{2}\left(\Omega^{*}\right)$, where $\varphi \in C_{0}^{\infty}(\Omega)$ and $r>0$. We apply the fact that $D u$ and $D v_{r}$ have the same singular parts. This implies

$$
\begin{aligned}
\int_{\Omega} & \sqrt{\mu^{2}+|\nabla u|^{2}} \mathrm{~d} x+\int_{\Omega}\left(\frac{\lambda}{2}|u|^{2}+f\left(u-u_{o}\right)\right) \mathrm{d} x \\
& \leq \int_{\Omega} \sqrt{\mu^{2}+|\nabla u-r \nabla \varphi|^{2}} \mathrm{~d} x+\int_{\Omega}\left(\frac{\lambda}{2}|u-r \varphi|^{2}+f\left(u-r \varphi-u_{o}\right)\right) \mathrm{d} x,
\end{aligned}
$$

and, after dividing by $r>0$,

$$
\begin{aligned}
\int_{\Omega} f \varphi \mathrm{d} x \leq & \frac{1}{r} \int_{\Omega}\left(\sqrt{\mu^{2}+|\nabla u-r \nabla \varphi|^{2}}-\sqrt{\mu^{2}+|\nabla u|^{2}}\right) \mathrm{d} x \\
& +\frac{\lambda}{2} \frac{1}{r} \int_{\Omega}\left(|u-r \varphi|^{2}-|u|^{2}\right) \mathrm{d} x .
\end{aligned}
$$

Letting $r \downarrow 0$ on the right-hand side, we deduce

$$
\begin{aligned}
\int_{\Omega} f \varphi \mathrm{d} x & \leq\left.\int_{\Omega} \frac{\partial}{\partial r}\right|_{r=0} \sqrt{\mu^{2}+|\nabla u-r \nabla \varphi|^{2}} \mathrm{~d} x+\left.\frac{\lambda}{2} \int_{\Omega} \frac{\partial}{\partial r}\right|_{r=0}|u-r \varphi|^{2} \mathrm{~d} x \\
& =-\int_{\Omega} \frac{\nabla u \cdot \nabla \varphi}{\sqrt{\mu^{2}+|\nabla u|^{2}}} \mathrm{~d} x-\lambda \int_{\Omega} u \varphi \mathrm{d} x .
\end{aligned}
$$

Note that it is allowed to differentiate under the integrals because in the first case, the derivative of the integral is dominated by $|\nabla \varphi| \in L^{1}(\Omega)$, and in the second integral, it is bounded by $2(|u|+|\varphi|)|\varphi| \in L^{1}(\Omega)$. In view of the definition of $z$, we have shown that

$$
\int_{\Omega}(\lambda u+f) \varphi \mathrm{d} x \leq-\int_{\Omega} z \cdot \nabla \varphi \mathrm{d} x
$$

holds true for every $\varphi \in C_{0}^{\infty}(\Omega)$. Since the same estimate holds with $-\varphi$ instead of $\varphi$, the opposite inequality holds as well. This proves $\operatorname{div} z=\lambda u+f$ in the distributional sense in $\Omega$.

Next, we use $w_{r}=u+r\left(u_{o}-u\right) \in \mathrm{BV}_{u_{o}}(\Omega) \cap L^{2}\left(\Omega^{*}\right)$, for $r>0$, as a comparison function for the minimizer $u$. Since $D^{s} w_{r}=(1-r) D^{s} u$, we obtain

$$
\begin{aligned}
& \int_{\Omega} \sqrt{\mu^{2}+|\nabla u|^{2}} \mathrm{~d} x+\left\|D^{s} u\right\|(\bar{\Omega})+\int_{\Omega}\left(\frac{\lambda}{2}|u|^{2}+f\left(u-u_{o}\right)\right) \mathrm{d} x \\
& \quad \leq \int_{\Omega} \sqrt{\mu^{2}+\left|\nabla w_{r}\right|^{2}} \mathrm{~d} x+(1-r)\left\|D^{s} u\right\|(\bar{\Omega})+\int_{\Omega}\left(\frac{\lambda}{2}\left|w_{r}\right|^{2}+(1-r) f\left(u-u_{o}\right)\right) \mathrm{d} x .
\end{aligned}
$$


Rearranging the terms and dividing by $r>0$, we deduce

$$
\begin{aligned}
& \left\|D^{s} u\right\|(\bar{\Omega})+\int_{\Omega} f\left(u-u_{o}\right) \mathrm{d} x \\
& \quad \leq \frac{1}{r} \int_{\Omega}\left(\sqrt{\mu^{2}+\left|\nabla w_{r}\right|^{2}}-\sqrt{\mu^{2}+|\nabla u|^{2}}\right) \mathrm{d} x+\frac{\lambda}{2} \frac{1}{r} \int_{\Omega}\left(\left|w_{r}\right|^{2}-|u|^{2}\right) \mathrm{d} x .
\end{aligned}
$$

Passing to the limit $r \downarrow 0$, we have

$$
\begin{array}{rl}
\| D^{s} & u \|(\bar{\Omega})+\int_{\Omega} f\left(u-u_{o}\right) \mathrm{d} x \\
& \leq\left.\int_{\Omega} \frac{\partial}{\partial r}\right|_{r=0} \sqrt{\mu^{2}+\left|\nabla w_{r}\right|^{2}} \mathrm{~d} x+\left.\frac{\lambda}{2} \int_{\Omega} \frac{\partial}{\partial r}\right|_{r=0}\left|w_{r}\right|^{2} \mathrm{~d} x \\
& =\int_{\Omega} \frac{\nabla u}{\sqrt{\mu^{2}+|\nabla u|^{2}}} \cdot\left(\nabla u_{o}-\nabla u\right) \mathrm{d} x+\lambda \int_{\Omega} u\left(u_{o}-u\right) \mathrm{d} x .
\end{array}
$$

Here, it is legitimate to differentiate under the integral because the derivative of the integrands are dominated by $\left|\nabla u_{o}-\nabla u\right| \in L^{1}(\Omega)$ and $2\left(|u|+\left|u_{o}\right|\right)\left|u_{o}-u\right| \in L^{1}(\Omega)$, respectively. By Young's inequality,

$$
\lambda \int_{\Omega} u\left(u_{o}-u\right) \mathrm{d} x \leq \frac{\lambda}{2} \int_{\Omega}\left(\left|u_{o}\right|^{2}-|u|^{2}\right) \mathrm{d} x .
$$

Combining the two preceding estimates and recalling the definition of $z$, we arrive at

$$
\begin{gathered}
\int_{\Omega} \frac{|\nabla u|^{2}}{\sqrt{\mu^{2}+|\nabla u|^{2}}} \mathrm{~d} x+\left\|D^{s} u\right\|(\bar{\Omega})+\int_{\Omega}\left(\frac{\lambda}{2}|u|^{2}+f\left(u-u_{o}\right)\right) \mathrm{d} x \\
\leq \int_{\Omega} z \cdot \nabla u_{o} \mathrm{~d} x+\frac{\lambda}{2} \int_{\Omega}\left|u_{o}\right|^{2} \mathrm{~d} x .
\end{gathered}
$$

For the first integrand on the left-hand side, we have the identity

$$
\frac{|\nabla u|^{2}}{\sqrt{\mu^{2}+|\nabla u|^{2}}}=\sqrt{\mu^{2}+|\nabla u|^{2}}-\mu \sqrt{1-|z|^{2}} .
$$

This corresponds to the equality case in (3.3). Thus (5.3) can be rewritten as

$$
\begin{gathered}
\int_{\Omega} \sqrt{\mu^{2}+|\nabla u|^{2}} \mathrm{~d} x+\left\|D^{s} u\right\|(\bar{\Omega})+\int_{\Omega}\left(\frac{\lambda}{2}|u|^{2}+f\left(u-u_{o}\right)\right) \mathrm{d} x \\
\leq \int_{\Omega} z \cdot \nabla u_{o} \mathrm{~d} x+\frac{\lambda}{2} \int_{\Omega}\left|u_{o}\right|^{2} \mathrm{~d} x+\mu \int_{\Omega} \sqrt{1-|z|^{2}} \mathrm{~d} x,
\end{gathered}
$$

which is the asserted estimate (5.2).

\subsection{A duality result for the total variation functional}

The following duality result will be applied in the proof of Theorem 5.1. More general duality results for problems with linear growth have been established in [7]. Here, we give a simple proof for a special case. The argument applies an approximation process given by Theorem 5.2. 
Theorem 5.3 Let $f \in W^{-1, \infty}(\Omega) \cap L^{2}(\Omega)$ with $\|f\|_{W^{-1, \infty}} \leq 1$ and $u_{o} \in W^{1,1}\left(\Omega^{*}\right) \cap$ $L^{2}\left(\Omega^{*}\right)$. Then we have

$$
\inf _{u \in \mathrm{BV}_{u_{o}}(\Omega) \cap L^{2}\left(\Omega^{*}\right)}\left(\|D u\|(\bar{\Omega})+\int_{\Omega} f\left(u-u_{o}\right) \mathrm{d} x\right)=\max _{z \in S_{f}^{\infty}(\Omega)} \int_{\Omega} z \cdot \nabla u_{o} \mathrm{~d} x,
$$

where $S_{f}^{\infty}(\Omega)=\left\{z \in L^{\infty}\left(\Omega, \mathbb{R}^{n}\right):\|z\|_{L^{\infty}} \leq 1\right.$ and $\left.\operatorname{div} z=f\right\}$.

Proof Let $u \in \mathrm{BV}_{u_{o}}(\Omega) \cap L^{2}\left(\Omega^{*}\right)$ and $z \in S_{f}^{\infty}(\Omega)$. By Lemma 3.4 and (3.2) we have

$$
\int_{\Omega} z \cdot \nabla u_{o} \mathrm{~d} x=(z, D u)_{u_{o}}(\bar{\Omega})+\int_{\Omega} f\left(u-u_{o}\right) \mathrm{d} x \leq\|D u\|(\bar{\Omega})+\int_{\Omega} f\left(u-u_{o}\right) \mathrm{d} x .
$$

Taking the supremum on the left-hand side and the infimum on the right, we infer

$$
\sup _{z \in S_{f}^{\infty}(\Omega)} \int_{\Omega} z \cdot \nabla u_{o} \mathrm{~d} x \leq \inf _{u \in \mathrm{BV}_{u_{o}}(\Omega) \cap L^{2}\left(\Omega^{*}\right)}\left(\|D u\|(\bar{\Omega})+\int_{\Omega} f\left(u-u_{o}\right) \mathrm{d} x\right) .
$$

In order to conclude the opposite inequality, we construct a minimizer of the functional

$$
\Psi_{\mu}(u):=\mathcal{A}_{u_{o}}^{(\mu)}(u)+\frac{\mu}{2} \int_{\Omega}|u|^{2} \mathrm{~d} x+\int_{\Omega} f_{\mu}\left(u-u_{o}\right) \mathrm{d} x
$$

in the space $\mathrm{BV}_{u_{o}}(\Omega) \cap L^{2}\left(\Omega^{*}\right)$ for $\mu \in(0,1)$, where $f_{\mu}:=(1-\mu) f$. Since every $u \in$ $\mathrm{BV}_{u_{o}}(\Omega) \cap L^{2}\left(\Omega^{*}\right)$ can be strictly approximated by functions $u_{i} \in\left(u_{o}+W_{0}^{1,1}(\Omega)\right) \cap L^{2}(\Omega)$, $i \in \mathbb{N}$, in the sense of Lemma 2.3, we have

$$
\begin{aligned}
\left|\int_{\Omega} f_{\mu}\left(u-u_{o}\right) \mathrm{d} x\right| & =\lim _{i \rightarrow \infty}\left|\int_{\Omega} f_{\mu}\left(u_{i}-u_{o}\right) \mathrm{d} x\right| \\
& \leq \lim _{i \rightarrow \infty}\left\|f_{\mu}\right\|_{W^{-1, \infty}}\left\|\nabla u_{i}-\nabla u_{o}\right\|_{L^{1}(\Omega)} \\
& \leq(1-\mu)\left(\|D u\|(\bar{\Omega})+\left\|\nabla u_{o}\right\|_{L^{1}(\Omega)}\right)
\end{aligned}
$$

for every $u \in \mathrm{BV}_{u_{o}}(\Omega) \cap L^{2}\left(\Omega^{*}\right)$. This implies the lower bound

$$
\begin{aligned}
\Psi_{\mu}(u) & \geq\|D u\|(\bar{\Omega})+\frac{\mu}{2}\|u\|_{L^{2}(\Omega)}^{2}-\left|\int_{\Omega} f_{\mu}\left(u-u_{o}\right) \mathrm{d} x\right| \\
& \geq \mu\|D u\|(\bar{\Omega})+\frac{\mu}{2}\|u\|_{L^{2}(\Omega)}^{2}-(1-\mu)\left\|\nabla u_{o}\right\|_{L^{1}(\Omega)}
\end{aligned}
$$

We deduce that $\Psi_{\mu}$ is coercive on the space $\mathrm{BV}_{u_{o}}(\Omega) \cap L^{2}\left(\Omega^{*}\right)$. Since, moreover, $\mathcal{A}_{u_{o}}^{(\mu)}$ is convex, the direct method of the calculus of variations yields the existence of a minimizer $u_{\mu} \in \mathrm{BV}_{u_{o}}(\Omega) \cap L^{2}\left(\Omega^{*}\right)$ of $\Psi_{\mu}$. We define the vector field

$$
z_{\mu}=\frac{\nabla u_{\mu}}{\sqrt{\mu^{2}+\left|\nabla u_{\mu}\right|^{2}}}
$$

where $\nabla u_{\mu}$ denotes the Lebesgue density of the absolutely continuous part of $D u_{\mu}$. Theorem 5.2 with $\lambda=\mu$ and $f$ replaced by $f_{\mu}$ implies that

$$
\operatorname{div} z_{\mu}=\mu u_{\mu}+f_{\mu} \quad \text { in } \Omega
$$


in the sense of distributions, as well as the estimate

$$
\begin{aligned}
& \mathcal{A}_{u_{o}}^{(\mu)}\left(u_{\mu}\right)+\int_{\Omega}\left(\frac{\mu}{2}\left|u_{\mu}\right|^{2}+f_{\mu}\left(u_{\mu}-u_{o}\right)\right) \mathrm{d} x \\
& \quad \leq \int_{\Omega} z_{\mu} \cdot \nabla u_{o} \mathrm{~d} x+\frac{\mu}{2} \int_{\Omega}\left|u_{o}\right|^{2} \mathrm{~d} x+\mu \int_{\Omega} \sqrt{1-\left|z_{\mu}\right|^{2}} \mathrm{~d} x .
\end{aligned}
$$

Since

$$
\mathcal{A}_{u_{o}}^{(\mu)}\left(u_{\mu}\right) \geq\left\|D u_{\mu}\right\|(\bar{\Omega}) \geq(1-\mu)\left\|D u_{\mu}\right\|(\bar{\Omega}),
$$

the left-hand side can be bounded from below in terms of the infimum in (5.5). More precisely, we have

$$
\begin{aligned}
& (1-\mu) \inf _{u \in \mathrm{BV}_{u_{o}}(\Omega) \cap L^{2}\left(\Omega^{*}\right)}\left(\|D u\|(\bar{\Omega})+\int_{\Omega} f\left(u-u_{o}\right) \mathrm{d} x\right) \\
& \leq \int_{\Omega} z_{\mu} \cdot \nabla u_{o} \mathrm{~d} x+\frac{\mu}{2} \int_{\Omega}\left|u_{o}\right|^{2} \mathrm{~d} x+\mu \int_{\Omega} \sqrt{1-\left|z_{\mu}\right|^{2}} \mathrm{~d} x
\end{aligned}
$$

for every $\mu \in(0,1)$. Since $\left\|z_{\mu}\right\|_{L^{\infty}} \leq 1$ for every $\mu \in(0,1)$, we can find a sequence $\mu_{i} \downarrow 0$ and a limit vector field $z_{*} \in L^{\infty}\left(\Omega, \mathbb{R}^{n}\right)$ with

$$
z_{\mu_{i}} \stackrel{*}{\rightarrow} z_{*} \text { weakly* in } L^{\infty}\left(\Omega, \mathbb{R}^{n}\right) \text {, as } i \rightarrow \infty .
$$

Using estimate (5.7) and the minimality of $u_{\mu}$, we infer the bound

$$
\begin{aligned}
\frac{\mu_{i}}{2}\left\|u_{\mu_{i}}\right\|_{L^{2}(\Omega)}^{2} & \leq \Psi_{\mu_{i}}\left(u_{\mu_{i}}\right)+\left(1-\mu_{i}\right)\left\|\nabla u_{o}\right\|_{L^{1}(\Omega)} \\
& \leq \Psi_{\mu_{i}}\left(u_{o}\right)+\left\|\nabla u_{o}\right\|_{L^{1}(\Omega)} \\
& \leq \int_{\Omega}\left(\sqrt{1+\left|\nabla u_{o}\right|^{2}}+\left|u_{o}\right|^{2}+\left|\nabla u_{o}\right|\right) \mathrm{d} x .
\end{aligned}
$$

This implies that the sequence of functions $\sqrt{\mu_{i}} u_{\mu_{i}}, i \in \mathbb{N}$, is bounded in $L^{2}(\Omega)$, and we get

$$
\mu_{i} \int_{\Omega} u_{\mu_{i}} \varphi \mathrm{d} x \leq \mu_{i}\left\|u_{\mu_{i}}\right\|_{L^{2}(\Omega)}\|\varphi\|_{L^{2}(\Omega)} \rightarrow 0
$$

as $i \rightarrow \infty$, for every $\varphi \in C_{0}^{\infty}(\Omega)$. We use this together with the convergence (5.10) to pass to the limit in (5.8), which implies $\operatorname{div} z_{*}=f$ in $\Omega$, in the sense of distributions. Since

$$
\left\|z_{*}\right\|_{L^{\infty}} \leq \liminf _{i \rightarrow \infty}\left\|z_{\mu_{i}}\right\|_{L^{\infty}} \leq 1
$$

we infer $z_{*} \in S_{f}^{\infty}(\Omega)$. Next, we use the convergence (5.10) to pass to the limit $i \rightarrow \infty$ in (5.9) and arrive at

$$
\begin{aligned}
& \inf _{u \in \mathrm{BV}_{u_{o}}(\Omega) \cap L^{2}\left(\Omega^{*}\right)}\left(\|D u\|(\bar{\Omega})+\int_{\Omega} f\left(u-u_{o}\right) \mathrm{d} x\right) \\
& \leq \int_{\Omega} z_{*} \cdot \nabla u_{o} \mathrm{~d} x \leq \sup _{z \in S_{f}^{\infty}(\Omega)} \int_{\Omega} z \cdot \nabla u_{o} \mathrm{~d} x \\
& \quad \leq \inf _{u \in \mathrm{BV}_{u_{o}}(\Omega) \cap L^{2}(\Omega)}\left(\|D u\|(\bar{\Omega})+\int_{\Omega} f\left(u-u_{o}\right) \mathrm{d} x\right) .
\end{aligned}
$$

For the last inequality, we recall (5.6). We conclude that we have an equality throughout, and in particular, the supremum above is attained. This completes the proof of (5.5). 


\subsection{Variational solutions are weak solutions}

In this subsection, we complete the proof of Theorem 5.1. To this end, assume that $u \in L_{w *}^{1}\left(0, T ; \mathrm{BV}_{u_{o}}(\Omega)\right) \cap C^{0}\left([0, T] ; L^{2}(\Omega)\right)$ is a variational solution of (1.1) with $\partial_{t} u \in$ $L^{2}\left(\Omega_{T}\right)$. Lemma 4.2 implies that the initial values are attained in the sense $u(0)=u_{o}(0)$ and that the slicewise variational inequality

$$
\|D u(t)\|(\bar{\Omega}) \leq \int_{\Omega \times\{t\}} \partial_{t} u(v-u) \mathrm{d} x+\|D v\|(\bar{\Omega})
$$

holds true for every $v \in \mathrm{BV}_{u_{o}(t)}(\Omega) \cap L^{2}\left(\Omega^{*}\right)$ and a.e. $t \in(0, T)$. For the proof of (3.7), we begin with the observation that the variational inequality (5.11) implies $\partial_{t} u \in L_{w *}^{\infty}\left(0, T ; W^{-1, \infty}(\Omega)\right)$ with

$$
\underset{t \in(0, T)}{\operatorname{ess} \sup }\left\|\partial_{t} u(t)\right\|_{W^{-1, \infty}(\Omega)} \leq 1 .
$$

In order to prove this claim, we consider an arbitrary $\varphi \in L^{1}\left(0, T ; W_{0}^{1,1}(\Omega)\right)$ and use $v=u(t)-\varphi(t)$ as a comparison function in the variational inequality (5.11). After integrating over $t \in(0, T)$, we obtain the bound

$$
\begin{aligned}
\iint_{\Omega_{T}} \partial_{t} u \varphi \mathrm{d} x \mathrm{~d} t & \leq \int_{0}^{T}(\|D u(t)-D \varphi(t)\|(\bar{\Omega})-\|D u(t)\|(\bar{\Omega})) \mathrm{d} t \\
& \leq \iint_{\Omega_{T}}|\nabla \varphi| \mathrm{d} x \mathrm{~d} t
\end{aligned}
$$

The preceding estimate implies

$$
\partial_{t} u \in\left[L^{1}\left(0, T ; W_{0}^{1,1}(\Omega)\right)\right]^{\prime}=L_{w *}^{\infty}\left(0, T ; W^{-1, \infty}(\Omega)\right),
$$

together with (5.12). Next, we note that the variational inequality (5.11) corresponds to a minimization property of $u(t) \in \mathrm{BV}_{u_{o}(t)}(\Omega) \cap L^{2}\left(\Omega^{*}\right)$. More precisely, for a.e. $t \in(0, T)$, the function $u(t)$ is a minimizer of the functional

$$
\Psi(v)=\|D v\|(\bar{\Omega})+\int_{\Omega \times\{t\}} \partial_{t} u\left(v-u_{o}\right) \mathrm{d} x
$$

in the space $\mathrm{BV}_{u_{o}(t)}(\Omega) \cap L^{2}\left(\Omega^{*}\right)$. In view of (5.12), Theorem 5.3 is applicable with the choice $f=\partial_{t} u(t) \in W^{-1, \infty}(\Omega) \cap L^{2}(\Omega)$, for a.e. $t \in(0, T)$. Theorem 5.3 implies

$$
\|D u(t)\|(\bar{\Omega})+\int_{\Omega \times\{t\}} \partial_{t} u\left(u-u_{o}\right) \mathrm{d} x=\max _{\tilde{z} \in S_{\partial_{t} u(t)}^{\infty}(\Omega)} \int_{\Omega \times\{t\}} \tilde{z} \cdot \nabla u_{o} \mathrm{~d} x
$$

for a.e. $t \in(0, T)$. Our next goal is to show that there is a vector field $z \in L^{\infty}\left(\Omega_{T}, \mathbb{R}^{n}\right)$ such that $z(t) \in L^{\infty}\left(\Omega, \mathbb{R}^{n}\right)$ realizes the maximum in (5.13) for a.e. $t \in(0, T)$. By definition of the maximum, for a.e. $t \in(0, T)$ we can choose a vector field $z_{*}(t) \in L^{\infty}\left(\Omega, \mathbb{R}^{n}\right)$ with $\left\|z_{*}(t)\right\|_{L^{\infty}(\Omega)} \leq 1$ and $\operatorname{div} z_{*}(t)=\partial_{t} u(t)$ in $\Omega$, such that

$$
\int_{\Omega \times\{t\}} z_{*} \cdot \nabla u_{o} \mathrm{~d} x=\max _{\tilde{z} \in S_{\partial_{t} u(t)}^{\infty}(\Omega)} \int_{\Omega \times\{t\}} \tilde{z} \cdot \nabla u_{o} \mathrm{~d} x .
$$

However, at this stage we can not rule out the possibility that $t \mapsto z_{*}(t)$ is not measurable. In this case, we need to replace $z_{*}$ by a measurable vector field $z \in L^{\infty}\left(\Omega_{T}, \mathbb{R}^{n}\right)$. To show the 
existence of such a vector field, we identify elements in $L^{\infty}\left(\Omega_{T}, \mathbb{R}^{n}\right)$ with bounded linear functionals on $L^{1}\left(\Omega_{T}, \mathbb{R}^{n}\right)$. Let us consider the subspace

$$
\mathcal{W}=\left\{r \nabla u_{o}+\nabla \varphi: r \in \mathbb{R}, \varphi \in C_{0}^{\infty}\left(\Omega_{T}\right)\right\} \subset L^{1}\left(\Omega_{T}, \mathbb{R}^{n}\right) .
$$

For a given element $V \in \mathcal{W}$, we choose $r \in \mathbb{R}$ and $\varphi \in C_{0}^{\infty}\left(\Omega_{T}\right)$ with $V=r \nabla u_{o}+\nabla \varphi$. For a.e. $t \in(0, T)$, we have

$$
\begin{aligned}
\int_{\Omega \times\{t\}} z_{*} \cdot V \mathrm{~d} x & =r \int_{\Omega \times\{t\}} z_{*} \cdot \nabla u_{o} \mathrm{~d} x+\int_{\Omega \times\{t\}} z_{*} \cdot \nabla \varphi \mathrm{d} x \\
& =r \max _{\tilde{z} \in S_{\partial_{t} u(t)}^{\infty}(\Omega)} \int_{\Omega \times\{t\}} \tilde{z} \cdot \nabla u_{o} \mathrm{~d} x-\int_{\Omega \times\{t\}} \partial_{t} u \varphi \mathrm{d} x .
\end{aligned}
$$

For the last identity, we used the choice of $z_{*}(t)$ according to (5.14) and the fact that $\operatorname{div} z_{*}(t)=\partial_{t} u(t)$. We observe that the maximum in the last line depends measurably on time because it coincides with a measurable function by (5.13), cf. Lemma 2.2. We conclude that the left-hand side of the preceding formula depends measurably on time as well. Moreover, because of $\left\|z_{*}(t)\right\|_{L^{\infty}} \leq 1$ for a.e. $t \in(0, T)$, we have

$$
\left|\int_{\Omega \times\{t\}} z_{*} \cdot V \mathrm{~d} x\right| \leq \int_{\Omega \times\{t\}}|V| \mathrm{d} x
$$

for a.e. $t \in(0, T)$. Therefore, we may define a linear functional

$$
\ell: \mathcal{W} \rightarrow \mathbb{R}, \quad V \mapsto \int_{0}^{T}\left(\int_{\Omega \times\{t\}} z_{*} \cdot V \mathrm{~d} x\right) \mathrm{d} t
$$

Integrating estimate (5.15) over time, we infer the bound

$$
|\ell(V)| \leq\|V\|_{L^{1}\left(\Omega_{T}\right)} \text { for every } V \in \mathcal{W} .
$$

This means that $\ell$ is a bounded linear functional on $\mathcal{W}$ with $\|\ell\|_{\mathcal{W}^{\prime}} \leq 1$. By the HahnBanach theorem, there exists an extension $L \in\left[L^{1}\left(\Omega_{T}, \mathbb{R}^{n}\right)\right]^{\prime}$ with $\left.L\right|_{\mathcal{W}}=\ell$ and

$$
\|L\|_{\left[L^{1}\left(\Omega_{T}, \mathbb{R}^{n}\right)\right]^{\prime}}=\|\ell\|_{\mathcal{W}^{\prime}} \leq 1
$$

The Riesz representation theorem yields a vector field $z \in L^{\infty}\left(\Omega_{T}, \mathbb{R}^{n}\right)$ with

$$
\|z\|_{L^{\infty}\left(\Omega_{T}\right)}=\|L\|_{\left[L^{1}\left(\Omega, \mathbb{R}^{n}\right)\right]^{\prime}} \leq 1
$$

and

$$
\iint_{\Omega_{T}} z \cdot V \mathrm{~d} x \mathrm{~d} t=L(V)=\int_{0}^{T}\left(\int_{\Omega \times\{t\}} z_{*} \cdot V \mathrm{~d} x\right) \mathrm{d} t
$$

for every $V \in \mathcal{W}$. We exploit this identity in two ways. First, we choose $V=\nabla \varphi \in \mathcal{W}$, where $\varphi \in C_{0}^{\infty}\left(\Omega_{T}\right)$, and deduce

$$
\iint_{\Omega_{T}} z \cdot \nabla \varphi \mathrm{d} x \mathrm{~d} t=\int_{0}^{T}\left(\int_{\Omega \times\{t\}} z_{*} \cdot \nabla \varphi \mathrm{d} x\right) \mathrm{d} t=-\iint_{\Omega_{T}} \partial_{t} u \varphi \mathrm{d} x \mathrm{~d} t,
$$


which means $\operatorname{div} z=\partial_{t} u$ in $\Omega_{T}$, in the sense of distributions. In view of (5.16), we infer $z(t) \in S_{\partial_{t} u(t)}^{\infty}(\Omega)$ for a.e. $t \in(0, T)$. Second, the choice $V=\nabla u_{o} \in \mathcal{W}$ in (5.17) implies

$$
\begin{aligned}
\iint_{\Omega_{T}} z \cdot \nabla u_{o} \mathrm{~d} x \mathrm{~d} t & =\int_{0}^{T}\left(\int_{\Omega \times\{t\}} z_{*} \cdot \nabla u_{o} \mathrm{~d} x\right) \mathrm{d} t \\
& =\int_{0}^{T}\left(\max _{\tilde{z} \in S_{\partial t u(t)}^{\infty}(\Omega)} \int_{\Omega \times\{t\}} \tilde{z} \cdot \nabla u_{o} \mathrm{~d} x\right) \mathrm{d} t \\
& \geq \iint_{\Omega_{T}} z \cdot \nabla u_{o} \mathrm{~d} x \mathrm{~d} t
\end{aligned}
$$

where we used (5.14) and the fact $z(t) \in S_{\partial_{t} u(t)}^{\infty}(\Omega)$ for a.e. $t \in(0, T)$. We deduce that the last inequality must be an identity, which implies

$$
\int_{\Omega \times\{t\}} z \cdot \nabla u_{o} \mathrm{~d} x=\max _{\tilde{z} \in S_{\partial_{t} u(t)}^{\infty}(\Omega)} \int_{\Omega \times\{t\}} \tilde{z} \cdot \nabla u_{o} \mathrm{~d} x
$$

for a.e. $t \in(0, T)$. Hence, we have found the desired vector field $z \in L^{\infty}\left(\Omega_{T}, \mathbb{R}^{n}\right)$ such that $z(t) \in S_{\partial_{t} u(t)}^{\infty}(\Omega)$ realizes the maximum in (5.13) for a.e. $t \in(0, T)$. Equation (5.13) implies the identity

$$
\|D u(t)\|(\bar{\Omega})+\int_{\Omega \times\{t\}} \partial_{t} u\left(u-u_{o}\right) \mathrm{d} x=\int_{\Omega \times\{t\}} z \cdot \nabla u_{o} \mathrm{~d} x
$$

for a.e. $t \in(0, T)$. For the proof of (3.7), it remains to replace $u_{o}$ by an arbitrary $v \in$ $\mathrm{BV}_{u_{o}(t)}(\Omega) \cap L^{2}\left(\Omega^{*}\right)$ in the preceding identity. This can be done with the help of Lemma 3.4. Since $\operatorname{div} z(t)=\partial_{t} u(t)$, Lemma 3.4 implies

$$
\int_{\Omega \times\{t\}} z \cdot \nabla u_{o} \mathrm{~d} x=(z(t), D v)_{u_{o}}(\bar{\Omega})+\int_{\Omega \times\{t\}} \partial_{t} u\left(v-u_{o}\right) \mathrm{d} x .
$$

Combining the two preceding identities, we arrive at

$$
\|D u(t)\|(\bar{\Omega})+\int_{\Omega \times\{t\}} \partial_{t} u(u-v) \mathrm{d} x=(z(t), D v)_{u_{o}}(\bar{\Omega})
$$

for a.e. $t \in(0, T)$ and every $v \in \mathrm{BV}_{u_{o}(t)}(\Omega) \cap L^{2}\left(\Omega^{*}\right)$, which is the assertion (3.7). Therefore, the function $u$ is a weak solution of (1.1) in the sense of Definition 3.5. This completes the proof of Theorem 5.1.

Acknowledgements The first author would like to thank Heikki Hakkarainen, Panu Lahti and Olli Saari for several useful discussions on this topic over the years.

Funding Open Access funding enabled and organized by Projekt DEAL.

Open Access This article is licensed under a Creative Commons Attribution 4.0 International License, which permits use, sharing, adaptation, distribution and reproduction in any medium or format, as long as you give appropriate credit to the original author(s) and the source, provide a link to the Creative Commons licence, and indicate if changes were made. The images or other third party material in this article are included in the article's Creative Commons licence, unless indicated otherwise in a credit line to the material. If material is not included in the article's Creative Commons licence and your intended use is not permitted by statutory regulation or exceeds the permitted use, you will need to obtain permission directly from the copyright holder. To view a copy of this licence, visit http://creativecommons.org/licenses/by/4.0/. 


\section{References}

1. Ambrosio, L., Fusco, N., Pallara, D.: Functions of Bounded Variation and Free Discontinuity Problems. Oxford Science Publications, Oxford (2000)

2. Andreu, F., Ballester, C., Caselles, V., Mazón, J.M.: Minimizing total variation flow. Differ. Integral Equ. 14(3), 321-360 (2001)

3. Andreu, F., Ballester, C., Caselles, V., Mazón, J.M.: The Dirichlet problem for the total variation flow. J. Funct. Anal. 180(2), 347-403 (2001)

4. Andreu, F., Caselles, V., Díaz, J.I., Mazón, J.M.: Some qualitative properties for the total variation flow. J. Funct. Anal. 188(2), 516-547 (2002)

5. Andreu, F., Caselles, V., Mazón, J.M.: Parabolic quasilinear equations minimizing linear growth functionals. Progress in Mathematics, 223, Birkhäuser Verlag, Basel (2004)

6. Anzellotti, G.: Pairings between measures and bounded functions and compensated compactness. Ann. Mat. Pura Appl. 135(4), 293-318 (1984)

7. Beck, L., Schmidt, T.: Convex duality and uniqueness for-minimizers. J. Funct. Anal. 268(10), 3061-3107 (2015)

8. Bögelein, V., Duzaar, F., Marcellini, P.: A time dependent variational approach to image restoration. SIAM J. Imaging Sci. 8(2), 968-1006 (2015)

9. Bögelein, V., Duzaar, F., Schätzler, L., Scheven, C.: Existence for evolutionary problems with linear growth by stability methods. J. Differ. Equ. 266(11), 7709-7748 (2019)

10. Bögelein, V., Duzaar, F., Scheven, C.: The obstacle problem for the total variation flow. Ann. Sci. Éc. Norm. Supér. 49(5), 1143-1188 (2016)

11. Bögelein,V., Duzaar, F., Scheven, C.: The total variation flow with time dependent boundary values. Calc. Var. Partial Differ. Equ. 55(4), Art. 108 (2016)

12. DiBenedetto, E.: Degenerate Parabolic Equations. Universitext. Springer, New York (1993)

13. DiBenedetto, E., Gianazza, U., Klaus, C.: A necessary and sufficient condition for the continuity of local minima of parabolic variational integrals with linear growth. Adv. Calc. Var. 10(3), 209-221 (2017)

14. Gianazza, U., Klaus, C.: p-parabolic approximation of total variation flow solutions. Indiana Univ. Math. J. 60(5), 1519-1550 (2019)

15. Górny, W., Mazón, J.M.: The Neumann and Dirichlet problems for the total variation flow in metric measure spaces. Preprint (2021)

16. Ionescu Tulcea, A., Ionescu Tulcea, C.: Topics in the theory of lifting. Ergebnisse der Mathematik und ihrer Grenzgebiete 48, Springer, New York (1969)

17. Lichnewsky, A., Temam, R.: Pseudosolutions of the time-dependent minimal surface problem. J. Differ. Equ. 30(3), 340-364 (1978)

18. Wieser, W.: Parabolic Q-minima and minimal solutions to variational flow. Manuscr. Math. 59(1), 63-107 (1987)

Publisher's Note Springer Nature remains neutral with regard to jurisdictional claims in published maps and institutional affiliations. 